\title{
Percutaneous Treatment of Aortic Coarctation in Young Adult Supported by Novel Fusion Imaging Technology
}

\author{
Iacopo Muraca ${ }^{1}$, Nazario Carrabba ${ }^{1}$, Giacomo Virgili ${ }^{2}$, Filippo Bruscoli ${ }^{2}$, Angela Migliorini ${ }^{1}$, \\ Matteo Pennesi ${ }^{1}$, Giulia Pontecorboli ${ }^{1,2}$, Niccolò Marchionni ${ }^{2}$, Francesco Bovenzi ${ }^{3}$, Pierluigi \\ Stefàno $^{2,4}$ and Renato Valenti ${ }^{1 *}$ \\ ${ }^{1}$ From the Interventional Cardiology Unit, Cardiovascular Department, Careggi University Hospital, Florence, Italy
}

${ }^{2}$ From the Department of Clinical and Experimental Medicine, University of Florence, Florence, Italy

${ }^{3}$ From the S.C. Cardiology, San Luca Hospital, Lucca, Italy

${ }^{4}$ From the Cardiac Surgery Unit, Cardiovascular Department, Careggi University Hospital, Florence, Italy

*Corresponding author: Renato Valenti, Interventional Cardiology, Careggi University Hospital, Florence, Italy; Largo Brambilla 3,

50141 Florence, Italy

\section{ARTICLE INFO}

Received: 幽 March 12, 2021

Published: March 22, 2021

Citation: Iacopo Muraca, Nazario Carrabba, Giacomo Virgili, Filippo Bruscoli, Renato Valenti, et al., Percutaneous Treatment of Aortic Coarctation in Young Adult Supported by Novel Fusion Imaging Technology. Biomed J Sci \& Tech Res 34(4)-2021. BJSTR. MS.ID.005585.

Keywords: Aortic Coarctation; Structural Percutaneous Interventions; Multimodality Imaging; Novel Techniques

\section{ABSTRACT}

Introduction: Aortic coarctation is one of the most frequent misdiagnosed congenital heart diseases and the most commonly missed fetal congenital heart disease. In adulthood the treatment of this pathology is primarily percutaneous.

Case Presentation: We present a case of a 37-year-old male with recent onset of heart failure symptoms due to misdiagnosed congenital aortic coarctation. The execution of a diagnostic multimodality imaging allowed to detect aortic coarctation, which after the multidisciplinary "heart team" discussion, received indication to percutaneous correction of the aortic defect. In the cath-lab, the use of novel digital techniques, integrating the angiographic images with previously acquired angio-CT, allowed a safe stent delivery with minimum amount of contrast injection.

Conclusion: Percutaneous treatment of aortic coarctation in young adult is safe and effective. Fusion imaging tools could be very useful to optimize procedural results and sparing contrast dye, suggesting their possible implementation in different percutaneous procedures.

Abbreviations: CHD: Congenital Heart Disease; CMR: Cardiac Magnetic Resonance; CoA: Coarctation of the Aorta; CT: Computer Tomography; ED: End Diastolic; EF: Ejection Fraction; ICU: Intensive Cardiologic Unit; LV: Left Ventricle; LVEDd: Left Ventricular End Diastolic Diameter; LVEDv: Left Ventricular End Diastolic Volume

\section{Introduction}

Coarctation of the aorta ( $\mathrm{CoA})$ accounts for $6 \%-8 \%$ of all congenital heart disease (CHD), but it is the most commonly missed fetal CHD diagnosis, withless than one-third of the cases been detected at prenatal screening. We describe a case of CoA in an adult patient we managed percutaneously.

\section{History of Presentation}

We present the case of a 37 years-old, who suffered from recent onset exertional dyspnoea and paroxysmal nocturnal dyspnoea, other than not previously reported lower limb claudicatio. At the out patient visit, elevated values of arterial pressure associated with severe left ventricular dysfunction (EF 0.30) were found, and 
the patient consequently hospitalized. The patient did not report any significant previous pathologies and did not take home-drugs. At the admission, physical examination showed a low anklebrachial index (0.59). EKG showed sinus rhythm with left bundle branch block; no arrhythmias were observed. The echocardiogram confirmed a severe reduction of left ventricular function in absence of segmental akinesia, other than dilated ascending aorta (42 $\mathrm{mm}$ ), and a peak gradient of $47 \mathrm{mmHg}$ at the distal tract of the aortic root. Cardiac magnetic resonance (CMR) was executed, and it showed mild left ventricular dilatation (LVEDv $112 \mathrm{ml} / \mathrm{m} 2$, LVESv $71 \mathrm{ml} / \mathrm{m} 2$ ), EF 0.37 with diffused hypokinesis, severe concentric hypertrophy of the left ventricle (LV mass index $133 \mathrm{~g} / \mathrm{m} 2$ ) associated with nonischemic pattern of late gadolinium enhancement (LGE). (Figure 1). The possible ischemic aetiology was excluded on the basis of the patient's low risk profile and typically non-ischemic fibrotic pattern of myocardial fibrosis on CMR examination. On the basis of clinical and instrumental findings a suspect of CoA was confirmed by a CTangiography of the thoracic and abdominal aorta showing a "septallike" isthmic coarctation (Figure 2). After diagnosis confirmation, the patient was transferred to our institution in order to select the best approach for CoA correction.

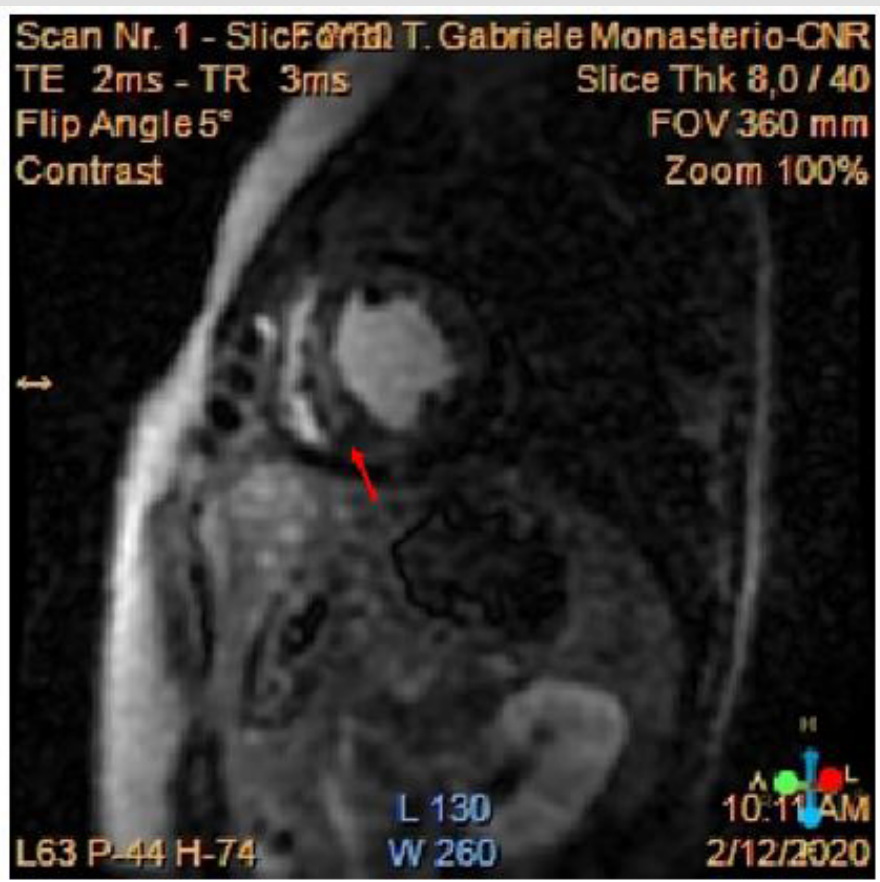

Figure 1: Cardiac MR showing a non-ischemic fibrotic pattern of LGE in particular at the level of the interventricular septum (red arrow).

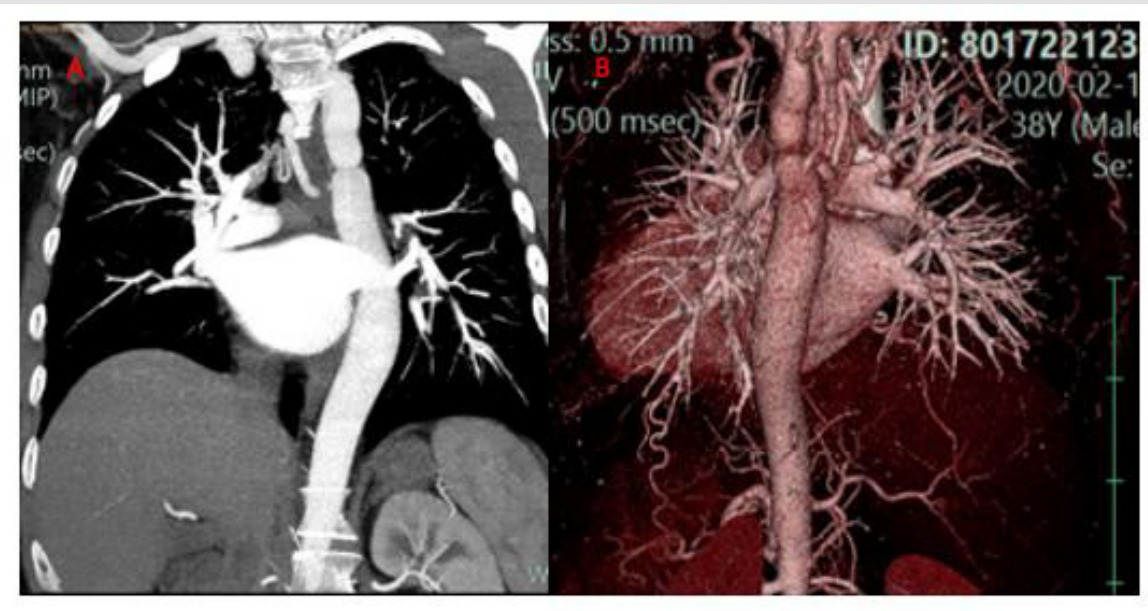

Figure 2:

A. Angio-CT images show a septum-like coarctation at the level of the isthmus;

B. $3 \mathrm{D}$ reconstruction. 


\section{Management}

After multidisciplinary "heart team" discussion, the patient received an indication to percutaneous correction of the aortic defect. The procedure was guided by multimodality imaging obtained by the fusion of cath-lab angiographic images with angioCT images. A $6 \mathrm{~F}$ right radial access and a $14 \mathrm{~F}$ echo-guided right femoral access were obtained. Coronary angiography confirmed the absence of coronary artery disease. Before proceeding with stent deployment, two $5 \mathrm{~F}$ pigtail were positioned on both sides of the coarctation in order to obtain a measurement of hemodynamic gradient between aortic root and abdominal aorta detecting a gradient of $46 \mathrm{mmHg}$. Successively, we started with crossing the aortic coarctation with a stiff hydrophilic guidewire and then advancing a multipurpose catheter in order to put in place an ultrastiff guide in ascending aorta. After that, we advance the covered stent delivery system and, guided by reference of vessel navigator system we deployed a covered stent especially designed for the treatment of vascular obstructions associated with congenital heart diseases: the Cheatham platinum (CP) stent (NuMED Inc, Hopkinton, New York, USA) in the coarctation site $(20 \times 39 \mathrm{~mm}$ in size and lenght) (Figure 3).

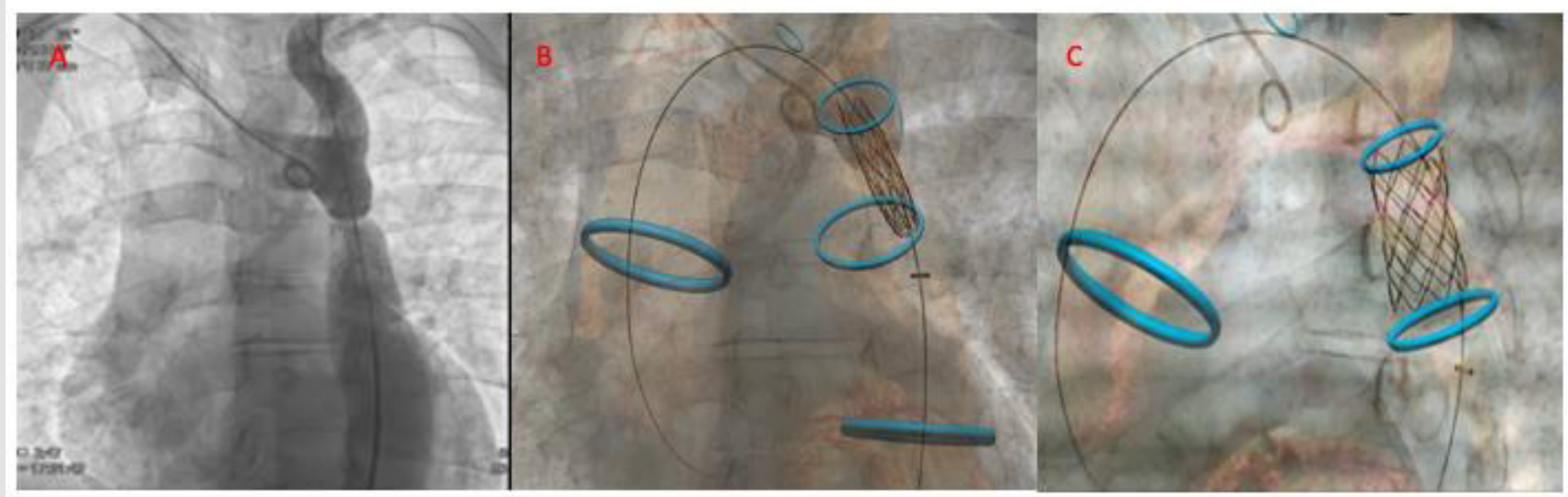

Figure 3: Fusion imaging technology: Different phase of stent delivery guided by angiographic and merged vessel navigator images

A. Positioning of the stent at the level of the aortic defect using angiographic and digital markers;

B. initial inflation of the inner balloon;

C. final stent positioning with sparing of left subclavian artery ostium.

\section{Figure 4:}

A. Hemodynamic evaluation of the upstream and downstream gradient through the aortic defect: separation of the curve in the thoracic and abdominal aorta with a maximum gradient of $55 \mathrm{mmHg}$;

B. overlapping of the curves with no gradient after stent delivery. 
Contrast injection confirmed the good positioning of the covered stent and no obstruction of left subclavian artery ostium. Before ending the procedure and closing the vascular accesses, a pigtail was inserted again at the level of the abdominal aorta to make a second measurement of the aortic gradient, not finding any pressure difference between the ascending aorta and the abdominal aorta (Figure 4). Fusion imaging supported good stent deployment, avoiding left subclavian artery obstruction. The volume of contrast dye used was $70 \mathrm{ml}$. No complications occurred during and after the procedure. Intra-hospital course was regular, and the patient was discharged in third postoperative day. At 6 months follow up, the patient showed a partial recover in LVEF (0.45) with normal arterial pressure value; no more exertional dyspnoea and lower limb claudicatio.

\section{Discussion}

Coarctation of the aorta (CoA) accounts for $6 \%-8 \%$ of all CHD, and it is the fifth most frequent one [1], but it is the most commonly missed fetal CHD diagnosis too, withless than one-third of the cases been detected at prenatal screening [2]. Coarctation of the aorta in adults accounts for $0.2 \%$ of all hypertension cases in adults [3]. CoA can be considered as a manifestation of a systemic arteriopathy [4]. It can present as a localized stenosis, or as a long, hypoplastic tract of the aorta, other than as an aortic arch interruption $[4,5]$. Typically, CoA is located near the ductal remnant and left subclavian artery [6]; unusually it occurs at the ascending aorta or abdominal aorta [7]. When the lesion is a discrete stenosis, it consists of a shelf in the posterolateral aortic wall opposite the (remnant of the) ductus arteriosus [5]. It is often associated with other cardiovascular lesions; the most frequent defect is bicuspid aortic valve (up to 85\%), ascending aortic aneurysm, sub/supraaortic stenosis, mitral valve disease or other CHD [4]. Extra-cardiac vascular anomalies have been reported in CoA patients and the most relevant are intracerebral aneurysms (2.5\% - 10\%) [8]. Our report is a case of aortic coarctation presenting in young male adult CoA presenting during adult life could be a missed case of native coarctation or more frequently a case of re-coarctation, following previous typically surgical treatment. CoA clinical presentation is variable, and depends on several factors, such as the site of coarctation or the presence of others associated lesions.

It can be asymptomatic or present with heart failure or endocarditis manifestations. Older patients may show symptoms such as headache, dizziness, tinnitus, abdominal angina, and exertional leg fatigue. The most common presenting finding is systemic hypertension [7]. In adult patients, differential diagnosis includes other causes of secondary arterial hypertension, possibly leading to hypertensive cardiomyopathy. CoA patients have a life expectancy of 35 years without treatment $[7,9]$, but treated ones have a shorter life expectancy too. Nowadays, patients who reach adolescence can survive up to 40 years after the initial repair if an adult CHD specialized center follow them up [10]. Close lifelong follow-up is mandatory, because of the important cardiovascular morbidity related to re-coarctation, descending aortic aneurysm, hypertension, and the other associated CHD $[7,8,10]$. First surgical repair happened in 1944 [11], then in 1983, Lock et al. performed the first percutaneous balloon angioplasty [12]. In 1991, a stent apposition repair was described, and finally, the first Covered Stent was used in 1999 [13]. Nowadays, the development of multimodality diagnostic imaging has allowed a careful pre-procedural planning of complex percutaneous structural interventions.

Furthermore, dedicated software could provide additional information to angiographic views, integrating pre- and procedural diagnostic images. Thus, this upgraded technology allows a higher intra-procedural accuracy, shorter procedural time with radiation sparing and lower amount of dye injection. This management could translate in better procedural outcomes. In our case, a multidisciplinary "heart team" decision was consistent with the last ESC guideline for the management of adult congenital heart disease, which indicates a percutaneous repair of aortic coarctation or recoarctation in adult patients when technically feasible [4]. The use of covered stent instead of uncovered one was preferred in order to reduce the possibility of complication after stent deployment.

\section{Conclusion}

Percutaneous treatment of aortic coarctation is a quite rare procedure, but it is associated with good safety and procedural success rate. In our procedure, the support of novel imaging technique played a key role for the deployment of the covered stent. The vessel navigator system allowed us to perform the procedure with a lower risk of left subclavian artery obstructing. Furthermore, the quality of imaging allowed a very low volume of contrast dye use.

\section{Conflict of Interest}

No conflict of interest.

\section{References}

1. Kenny D, Hijazi ZM (2011) Coarctation of the aorta: From fetal life to adulthood. Cardiol J 18(5): 487: 495.

2. Liberman RF, Getz KD, Lin AE, Higgins CA, Sekhavat S, et al. (2014) Delayed Diagnosis of Critical Congenital Heart Defects: Trends and Associated Factors. Pediatrics 134(2): e373-e381.

3. Prisant LM, Mawulawde K, Kapoor D, Joe C (2004) Coarctation of the aorta: A secondary cause of hypertension. J Clin Hypertens Greenwich Conn 6(6): 347-352.

4. Baumgartner H, De Backer J, Babu Narayan SV, Budts W, Chessa M, et al. (2020) 2020 ESC Guidelines for the management of adult congenital heart disease. Eur Heart J 47(6).

5. Zipes DP, Libby P, Bonow RO, Mann DL, Tomaselli GF (I2019) Braunwald's heart disease: A textbook of cardiovascular medicine. (11th Edn.). international, Elsevier, Philadelphia, PA, USA, pp. 1944. 
6. Stout KK, Daniels CJ, Aboulhosn JA, Bozkurt B, Broberg CS, et al. (2019) 2018 AHA/ACC Guideline for the Management of Adults with Congenital Heart Disease. J Am Coll Cardiol 139(14): e81-192.

7. Cribbs MG (2020) Coarctation: A Review. US Cardiol Rev 13(2): 99-104.

8. Dijkema EJ, Leiner T, Grotenhuis HB (2017) Diagnosis, imaging and clinical management of aortic coarctation. Heart 103(15): 1148-1155.

9. Torok RD (2015) Coarctation of the aorta: Management from infancy to adulthood. World J Cardiol 7(11): 765-775.

\section{ISSN: 2574-1241}

DOI: 10.26717/BJSTR.2021.34.005585

Renato Valenti. Biomed J Sci \& Tech Res

(C) This work is licensed under Creative

Submission Link: https://biomedres.us/submit-manuscript.php
10. Choudhary P, Canniffe C, Jackson DJ, Tanous D, Walsh K, et al. (2015) Late outcomes in adults with coarctation of the aorta. Heart 101(15): 1190-1195.

11. Crafoord C, Nylin G (1945) Congenital Coarctation Of the Aorta And Its Surgical Treatment. J Thorac Surg 14(5): 347-361.

12. O'Laughlin MP, Perry SB, Lock JE, Mullins CE (1991) Use of endovascular stents in congenital heart disease. Circulation 83(6): 1923-1939.

13. Gunn J, Cleveland T, Gaines P (1999) Covered stent to treat co-existent coarctation and aneurysm of the aorta in a young man. Heart 82(3): 351.

$\begin{array}{ll}\text { BIOMEDICAL } & \text { Assets of Publishing with us } \\ \text { RESEARCHES } & \text { - Global archiving of articles } \\ \text { - Immediate, unrestricted online access }\end{array}$

